\title{
COMMENTARY
}

\section{Pragmatic fluid optimization in high-risk surgery patients: when pragmatism dilutes the benefits}

\author{
Daniel A Reuter* \\ See related research by Cuthbertson et al., http://ccforum.com/content/15/6/R296
}

\begin{abstract}
There is increasing evidence that hemodynamic optimization by fluid loading, particularly when performed in the early phase of surgery, is beneficial in high-risk surgery patients: it leads to a reduction in postoperative complications and even to improved long-term outcome. However, it is also true that goaldirected strategies of fluid optimization focusing on cardiac output optimization have not been applied in the clinical routine of many institutions. Reasons are manifold: disbelief in the level of evidence and on the accuracy and practicability of the required monitoring systems, and economics. The FOCCUS trial examined perioperative fluid optimization with a very basic approach: a standardized volume load with $25 \mathrm{ml} / \mathrm{kg}$ crystalloids over 6 hours immediately prior to scheduled surgery in high-risk patients. The hypothesis was that this intervention would lead to a compensation of preoperative fluid deficit caused by overnight fasting, and would result in improved perioperative fluid homeostasis with less postoperative complications and earlier hospital discharge. However, the primary study endpoints did not improve significantly. This observation points towards the facts that: firstly, the differentiation between interstitial fluid deficit caused by fasting and intravascular volume loss due to acute blood loss must be recognized in treatment strategies; secondly, the type of fluid replacement may play an important role; and thirdly, protocolized treatment strategies should also always be tailored to suit the patients' individual needs in every individual clinical situation.
\end{abstract}

*Correspondence: dreuter@uke.de

Department of Anesthesiology, Center of Anesthesiology and Intensive Care Medicine, Hamburg-Eppendorf University Medical Center, Martinistraße 52, 20246 Hamburg, Germany
The article by Cuthbertson and colleagues deals with an important topic these days in perioperative anesthesia and intensive care [1]. Numerous clinical studies published in recent years seem to fit together like pieces of a puzzle, with the same overall message: hemodynamic optimization by fluid loading, particularly when performed in the early phase of surgery, is beneficial in highrisk surgery patients, and may even improve long-term outcome [2-4]. This outcome indeed sounds very attractive: a significant reduction in morbidity and even mortality by intravascular fluid therapy - oriented towards the physiological goals of cardiac output and oxygen delivery [5].

Recent advances in the development of less invasive or even completely non-invasive monitoring techniques also seem to provide reasonable or even better alternatives to the pulmonary artery catheter. The pulmonary artery catheter has now lost its place as the routine monitor of choice in perioperative medicine - if it even had this status in the operating rooms outside cardiac surgery [6]. There are still indications for use of the pulmonary artery catheter, but these have become scarce $[7,8]$. However, there is one major pitfall: as many had presumed, and as a recent survey of North American and European anesthesiologists confirmed, goal-directed strategies of fluid optimization focusing on cardiac output optimization have not been implemented routinely [9]. The reasons for this are manifold, of course. For some, the data from clinical studies are still not strong enough; some are not convinced by the accuracy or practicability of the monitoring equipment used to measure cardiac output or fluid responsiveness; many still avoid the potential extra costs for the necessary monitoring equipment due to a lack of reimbursement; and some may simply not be motivated enough to change their current clinical practice.

So how do we resolve this problem in order to give our patients the therapy they deserve? Option one is goaldirected treatment strategies supported by algorithms in combination with adequate advanced monitoring becoming routine in the operating rooms and ICUs - but this also means an increase in complexity and in the level of 
difficulty of hemodynamic perioperative care. Option two is for us to find another, simpler and more pragmatic, intervention in connection with a simpler protocol for all patients, which is as efficient as option one.

This latter option was basically the idea of the FOCCUS study [9]. The investigators assumed that optimized fluid management meant overall more fluid perioperatively, as indeed some of the published studies in this field have apparently reported. Furthermore, they assumed that our patients were already in fluid deficit prior to surgery, due to the classic and common practice in many places of nil by mouth after midnight, despite other clear recommendations $[10,11]$. The approach taken by the authors was to give a standardized amount of intravenous fluid (here $25 \mathrm{ml} / \mathrm{kg}$ Ringers' solution) in the last 6 hours prior to surgery, so fluid optimization was not just early, as proclaimed, but very early, very pre-emptive - and very pragmatic, and indeed very simple. Simplistic?

The results of this study are at first sight disappointing. There was no significant difference in the primary outcome - the number of hospital days following surgery nor in the secondary outcome variables - postoperative morbidity and mortality. A very complex analysis of costeffectiveness of the proposed intervention, however, demonstrated a positive effect. But why the clinical failure? What we have learned from the numerous hemodynamic optimization studies in the past is that all three aspects - timing, goals, and type of intervention must be set correctly. Timing should be early, when the deficit occurs. Preoperative fasting leads to a preoperative fluid deficit, but not primarily to an intravascular fluid deficit [12]. Reducing such a preoperative fluid deficit makes physiological sense, but this could be reached by simpler methods - namely by encouraging patients to drink clear fluids up to 2 hours before elective surgery, as recommended, and not, as is still the reality in many hospitals, with much longer fasting times [10]. Moreover, drinking water would be even more cost-effective than using intravenous Ringers' lactate solutions. But the intravascular fluid loss due to intraoperative bleeding or due to fluid shift out of the intravascular compartment caused by inflammation leads to much more significant fluid loss because this intravascular fluid loss immediately leads to low cardiac output and reduced oxygen delivery. These complications, however, occur in each patient at different time points and to different degrees during and immediately after surgery. Here, differential diagnosis must be performed, and tailored therapy must be initiated - immediately.

Defining the correct goals is another important discussion. Supranormal goals for oxygen delivery and cardiac output do not seem to be the answer $[11,13]$. Whether the key concept is optimization of the stroke volume and cardiac output by volume loading alone or with the addition of catecholamines, or whether it is the use of parameters of fluid responsiveness in combination with cardiac output monitoring, or one parameter alone, is still a matter of lively debate $[1,2,14]$. This also concerns the question of fluid type - crystalloid or colloid - which should also be tailored to the clinical situation. But it seems that we have the great opportunity of improving therapies for the sake of our patients by optimizing hemodynamics, and in particular the fluid status, prior to, during, and after surgery. Algorithmic, protocolized treatment strategies do seem to help. But protocolized, algorithmic supported care does not mean we leave behind the individualized care of every individual patient and every single clinical situation.

Are simple approaches too pragmatic? Actually, pragmatism by definition centers on the linking of practice and theory, and describes a process where theory is extracted from practice, and applies back to practice to form what is called in the words of John Dewey 'intelligent practice' [15]. Standardized, body-weightadapted preoperative intravenous fluid loading is not pragmatic. We have the knowledge, we have the evidence, and we have the technology to be pragmatic. So let us do it. Our patients deserve it.

\section{Competing interests}

The author declares that he has no competing interests.

Published: 31 January 2012

\section{References}

1. Cuthbertson B, Campbell M, Stott SA, Elders A, Hernandez R, Boyers D, Norrie J, Kinsella J, Brittenden J, Cook J, Rae D, Cotton SC, Alcorn D, Addison J, Grant A; FOCCUS study group: A pragmatic multi-centre randomised controlled trial of fluid loading in high-risk surgery patients undergoing major elective surgery - the FOCCUS study. Crit Care 2011, 15:R296.

2. Hamilton MA, Cecconi M, Rhodes A: A systematic review and meta-analysis on the use of preemptive hemodynamic intervention to improve postoperative outcomes in moderate and high-risk surgical patients. Anesth Analg 2011, 112:1392-1402.

3. Gurgel ST, do Nascimento $P$, Jr: Maintaining tissue perfusion in high-risk surgical patients: a systematic review of randomized clinical trials. Anesth Analg 2011, 112:1384-1391.

4. Rhodes A, Cecconi M, Hamilton M, Poloniecki J, Woods J, Boyd O, Bennett D, Grounds RM: Goal-directed therapy in high-risk surgical patients: a 15-year follow-up study. Intensive Care Med 2010, 36:1327-1332.

5. Strunden MS, Heckel K, Goetz AE, Reuter DA: Perioperative fluid and volume management: physiological basis, tools and strategies. Ann Intensive Care 2011, 1:2.

6. Reuter DA, Huang C, Edrich T, Shernan SK, Eltzschig HK: Cardiac output monitoring using indicator-dilution techniques: basics, limits, and perspectives. Anesth Analg 2010, 110:799-811.

7. Trepte CJ, Eichhorn V, Haas SA, Richter HP, Goepfert MS, Kubitz JC, Goetz AE, Reuter DA: Thermodilution-derived indices for assessment of left and right ventricular cardiac function in normal and impaired cardiac function. Crit Care Med 2011, 39:2106-2112.

8. Boyd $\mathrm{JH}$ : Globalization of ejection fraction: the pulmonary artery catheter has been outsourced [editorial]. Crit Care Med 2011, 39:2192.

9. Cannesson M, Pestel G, Ricks C, Hoeft A, Perel A: Hemodynamic monitoring and management in patients undergoing high risk surgery: a survey among north American and European anesthesiologists. Crit Care 2011; 15:R197.

10. Smith I, Kranke P, Murat I, Smith A, O'Sullivan G, Søreide E, Spies C, in't Veld B; 
European Society of Anaesthesiology: Perioperative fasting in adults and children: guidelines from the European Society of Anaesthesiology. Eur J Anaesthesiol 2011, 28:556-569.

11. Hayes MA, Timmins AC, Yau EH, Palazzo M, Hinds CJ, Watson D: Elevation of systemic oxygen delivery in the treatment of critically ill patients. N Engl J Med 1994, 330:1717-1722.

12. Jacob M, Chappell $D$, Conzen P, Finsterer U, Rehm M: Blood volume is normal after pre-operative overnight fasting. Acta Anaesthiol Scand 2008, 52:522-529.

13. Gattinoni L, Brazzi L, Pelosi P, Latini R, Tognoni G, Pesenti A, Fumagalli R: A trial of goal-oriented hemodynamic therapy in critically ill patients. $\mathrm{SvO}_{2}$ Collaborative Group. NEngl J Med 1995, 333:1025-1032.
14. Parker SJ, Boyd O: Haemodynamic optimization: are we dynamic enough? Crit Care 2011, 15:1003.

15. Dewey J: The Quest for Certainty: A Study of the Relation of Knowledge and Action. Minton, Balch, New York: The Gifford Lectures; 1929.

doi:10.1186/cc10604

Cite this article as: Reuter DA: Pragmatic fluid optimization in high-risk surgery patients: when pragmatism dilutes the benefits. Critical Care 2012, 16:106. 